\title{
Using Cell Free DNA Reference Standards to Evaluate the Analytical Performance of Circulating Tumor DNA Testing and Solid Organ Transplant Health Surveillance
}

Hadas Amit', Shen Wei', Javier Armisen-Garrido', Bernice Edgeworth', Robert Woodward², John J. Sninsky², Colin Barker', and Karin Schmitt ${ }^{1}$

${ }^{1}$ Horizon Discovery limited, 7100 Cambridge Research Park, Waterbeach, Cambridge CB25 9TL, United Kingdom. ${ }^{2}$ CareDx Inc, 3260 Bayshore Blvd, Brisbane, CA 94005, United States

\section{Introduction}

Development of new liquid biopsy techniques is rapidly evolving and includes methods ranging from broad sequencing to targeted molecular assays, with potential utility as companion diagnostics, in disease monitoring, evaluating efficacy of treatment, and perhaps in the future early cancer detection. Yet, the development of novel platforms is challenging due to the low abundance of cell free DNA (cfDNA) in human fluids and the difficulty in obtaining a sustainable source of patient-relevant material. Thus, translating the potential of a 'liquid biopsy' into the clinic requires precise and accurate technologies to enable sensitive and specific measurements. Moreover, there is a need to ensure clinical relevance, particularly where limitations are identified within a workflow approach, sampling method, or limit of detection. As with any clinical protocol, sample handling of cfDNA will require novel technologies through sound quality assurance and control methodologies, including comprehensive validation activities.

To meet these challenges, we present the design and application of fit-for-purpose cfDNA Reference Standards to evaluate the analytical performance of DNA assays for cell free circulating tumor DNA for oncology and 'transgenome' for solid organ transplantation (SOT). In SOT, recent work has informed clinically relevant ranges (DeVlaminck et al, Science Transl Med 2014; Gielis et al, Am J Transp 2015).

\section{Development of cfDNA Reference Standards}

Utilising proprietary genome editing technology, Horizon's Diagnostics division has developed a range of best-in-class, genetically defined cfDNA Reference Standards. Quantitative mixtures of genomic DNA extracted from highly validated isogenic cell lines were fragmented by mechanical shearing (Covaris S220) and validated using the Tapestation system (Agilent Tech.) for DNA fragmentation and digital PCR for absolute quantification of mutant and wild type variants.

\section{Product Features}

- DNA fragmented to a size distribution comparable to circulating DNA extracted from plasma.

- Accurate reference material at allelic frequencies down to $0.1 \%$ across a range of markers as well as true wild type control material.

\begin{tabular}{|c|c|}
\hline BRAF V600E & EGFR T790M \\
\hline EGFR L858R & EGFR $\triangle E 746-750$ \\
\hline \multicolumn{1}{|c|}{ EGFR V769_D770insASV } \\
\hline NRAS A59T & NRAS Q61K \\
\hline KRAS G12D & PI3K E545K \\
\hline
\end{tabular}

Key Product Specification

- Format: DNA

KRAS G12D

PI3K E545K

- Concentration: $40 \mathrm{ng} / \mathrm{\mu l}$ (verified by Qubit dsDNA BR assay)

- Fragmentation: DNA fragmented to an average size of 160bp (Figure 1).

- Allelic frequency: mutant frequency verified on the Bio-Rad QX100 system (Figures 2 and 3).

- Intended Use: For Routine Validation (Research Use Only) 


\section{Evaluating the Analytical Performance of a Cell-free DNA Assay for Solid Organ Transplantation (SOT)}

Multiple reference standard panels were constructed with a 'donor' cell line (RKO with EGFR T790M) and 'recipient' cell lines with different genotypes (SW48, HCT116, and HCC-78). The reference standards were used to characterize the analytical performance of an assay designed to determine the level of donor-derived cfDNA (dd-cfDNA) present in transplant recipient plasma as a percentage of the total plasma cfDNA. This assay uses targeted amplification of 266 carefully selected SNPS, followed by next-generation sequencing (NGS), to calculate the \%dd-cfDNA.

Using digital PCR to quantify trace amounts of DNA in the mixtures, as in Figures $2 \& 3$, the assembly of accurate reference material was confirmed, even at allelic frequencies down to $0.25 \%$ across a range of genotypes. The \%dd-cfDNA assay for SOT independently identifies the spikein concentration of the genome with high linearity and accuracy (Figure 4) despite low 'donor' copies. At 8ng input mass (approximating low cfDNA from $1.25 \mathrm{ml}$ plasma), there are approximately 11 copies of 'donor' at the 0.4\% level. In addition, the standards were used to characterize the limit of detection and quantification of the dd-cfDNA NGS assay for solid organ transplant health surveillance.

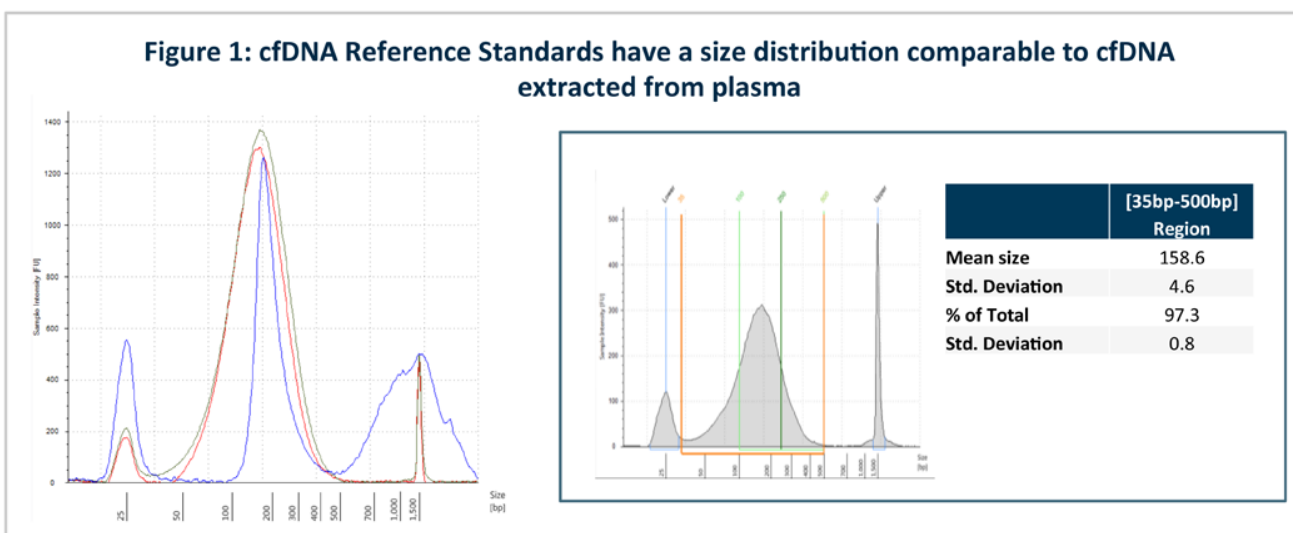

Cell free DNA extracted from human plasma (left panel, blue) and cfDNA Reference Standards (left panel, green and red, and right panel) analyzed for size distribution by High Sensitivity D1000 and D1000 ScreenTape assay on the Tapestation system, demonstrating that the cfDNA Reference Standards have an average size of 160bp approximating cell free DNA extracted from plasma.

Figure 2: Allelic frequency verification of NRAS Q61K down to $0.1 \%$ mutant frequency in cfDNA Reference Standards
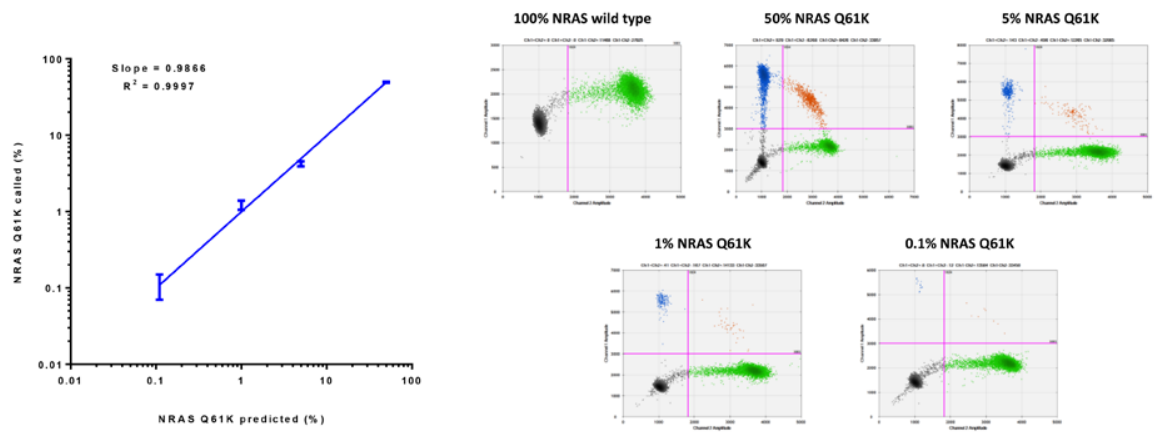

Left: Serial dilution of NRAS Q61K and mutant calling on cfDNA Reference standards quantified by droplet digital PCR, demonstrating the extreme accuracy of these standards. Data represents four technical replicates with 50ng of template DNA per well. Right: 2D plots for fragmented wild type (SW48 Parental) NRAS Q61K mutant samples demonstrating excellent assay performance on fragmented DNA.

Figure 3: Allelic frequency verification of EGFR $\triangle E 746-A 750$ down to $0.1 \%$ mutant frequency in cfDNA Reference Standards
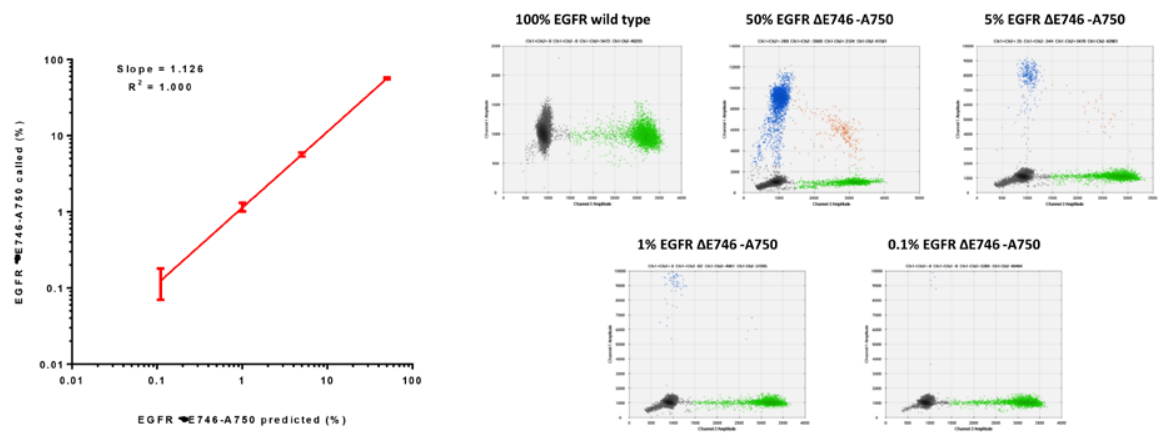

Left: Serial dilution of EGFR $\triangle E 746-A 750$ and mutant calling on cfDNA Reference standards quantified by droplet digital, demonstrating the extreme accuracy of these standards PCR. Data represents four technical replicates with 100ng of template DNA per well. Right: 2D plots for fragmented wild type (RKO Parental) EGFR $\triangle E 746-A 750$ mutant samples, demonstrating excellent assay performance on fragmented DNA. 
Figure 4: Independent Measurements Confirm Spike-In Accuracy of Reference Standards

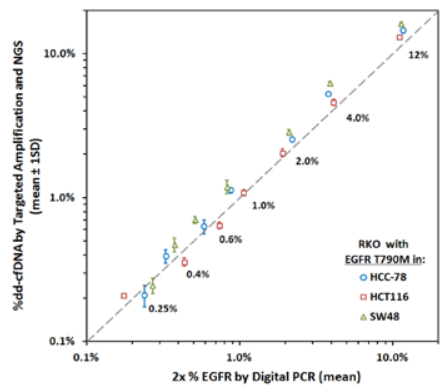

Three panels were created independently from DNA from a single 'donor' and three 'recipient' cell lines. Targeted amplification followed by NGS was used to determine the percent of 'donor' genome present ( $y$-axis). Digital PCR targeting the mutation (EGFR T790M) present in a single copy in the 'donor' genome, was multiplied by two to represent the genome percentage ( $x$-axis).

Digital PCR and NGS verification using cfDNA Reference Standards demonstrate these assays are accurate and reproducible across a range of allelic frequencies.
Figure 5: Reproducibility of the Targeted Amplification \& NGS Assay Determined by Reference Standards

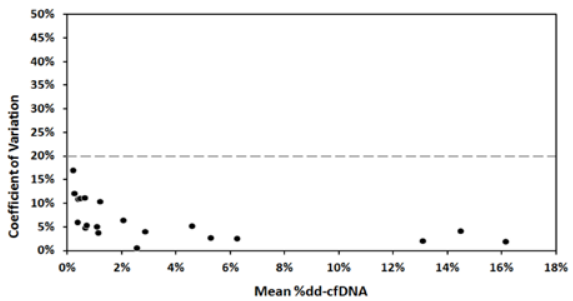

Targeted amplification followed by NGS Sequencing was used to determine the amount of RKO cell DNA spiked into DNA from three different cell lines. Four replicates run on different days by different operators were used to determine the coefficient of variation $(\mathrm{CV}=\mathrm{SD} / \mathrm{mean} \times 100)$ for each of 7 levels on 3 panels. The Lower Limit of Quantification (LLOQ) was defined as the lowest level of \%dd-cfDNA measured at which the CV was less than $20 \%$. All replicate sets were less than $20 \%$ CV.

NGS verification using cfDNA Reference Standards demonstrated these assays are reproducible across a range of allelic frequencies. The LLOQ of this assay determined using cfDNA Reference Standards is $0.4 \%$.

Figure 6: Performance Characteristics of the Targeted Amplification \& NGS Assay for \%dd-cfDNA Map to the Distribution of \%dd-cfDNA in Plasma from Heart and Kidney Transplant Recipients

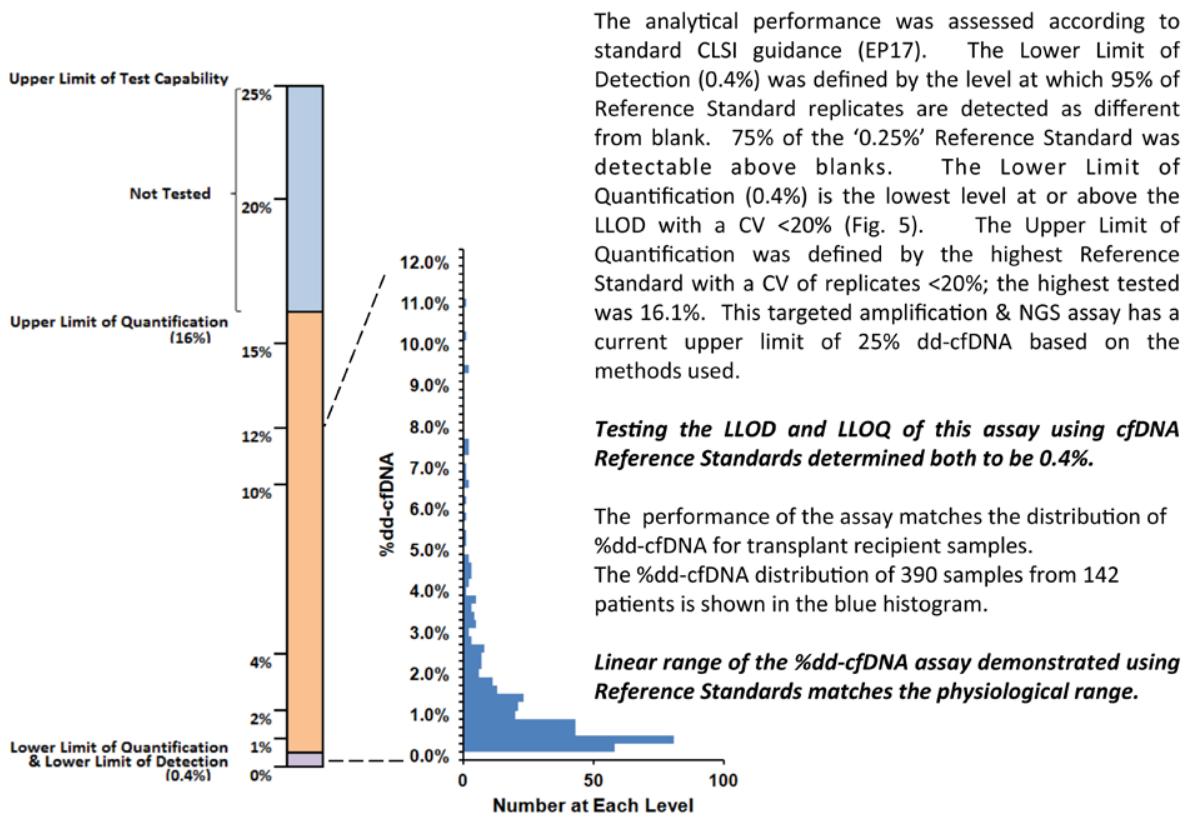

\section{Summary}

This study presents a proof-of-concept for the application of cfDNA Reference Standards in defining analytical performance of cfDNA testing across PCR and NGS platforms, enabling the development of different molecular profiling assays, routine cell free DNA testing, and cross-platform comparison. The flexibility and clinical relevance of engineered cell lines provides a versatile tool for assay optimization and validation. 